\title{
Assessment of odontogenic changes in computed tomography of patients with chronic sinusitis
}

Paulina Joanna Czarnecka ${ }^{1}$, Tomasz Zatoński², Hanna Gerber ${ }^{3}$, Monika Rutkowska ${ }^{1}$

\author{
${ }^{1}$ Otolaryngology, Head and Neck Surgery Clinic, $4^{\text {th }}$ Military Clinical Hospital, Wroclaw, \\ Poland \\ ${ }^{2}$ Department and Clinic of Otolaryngology, Head and Neck Surgery, Wroclaw Medical \\ University, Poland \\ ${ }^{3}$ Department and Clinic of Maxillofacial Surgery, Wroclaw Medical University, Poland
}

Submitted: 29 March 2020; Accepted: 1 February 2021

Online publication: 21 March 2021

Arch Med Sci

DOI: https://doi.org/10.5114/aoms/132968

Copyright @ 2021 Termedia \& Banach

\section{Abstract}

Introduction: Chronic sinusitis can be caused by both laryngological and dental factors. The frequency of odontogenic sinusitis (OS) in the last decades has been increasing and seems to be underrated. The unique developmental and microbiological factors causing OS require a different therapeutic approach.

Material and methods: This study evaluated tomography examinations of 500 patients with a clinical diagnosis of chronic sinusitis. The patients were referred by laryngologists, neurologists, and maxillofacial surgeons. The scans were reanalyzed in view of the presence of odontogenic and laryngological pathologies.

Results: Among the 500 patients, $19.6 \%$ showed no inflammatory changes in the mucosa of the paranasal sinuses. All patient groups had numerous teeth missing, ranging from $27.3 \%$ to $33.2 \%$. The most common odontogenic pathologies were periapical changes $(28.8 \%)$ and the presence of teeth after improper endodontic treatment (24.2\%). In the group in question dental implants (0.4\%) and maxillary sinus augmentation (2.8\%) were marginal etiological factors.

Conclusions: Computed tomography allows a thorough assessment of odontogenic changes. Obstruction of the ostiomeatal complex does not have direct influence on OS development. $43.2 \%$ of patients with chronic sinusitis have OS. It can be diagnosed in $50.8 \%$ of isolated right, $39.0 \%$ of isolated left and $57.8 \%$ of bilateral maxillary sinusitis patients. The results of this study can be used by dentists, maxillofacial surgeons and otolaryngologists to improve the standard of diagnosis and treatment in cases of chronic odontogenic sinusitis.

Key words: dental implants, cone-beam computed tomography, periimplantitis, odontogenic cyst, maxillary sinusitis.

\section{Introduction}

Chronic sinusitis is inflammation of the mucous membranes of the nose or of the paranasal sinuses; the inflammation is linked to allergic, bacterial, and fungal factors as well as conditioned anatomical determinants [1].

Chronic sinusitis is defined as the presence of two or more symptoms lasting more than 12 weeks, one of which must be nasal congestion or

\author{
Corresponding author: \\ Paulina Joanna Czarnecka \\ Otolaryngology, Head and \\ Neck Surgery Clinic \\ $4^{\text {th }}$ Military Clinical Hospital \\ Wroclaw, Poland \\ E-mail: paulina.j.czarnecka@ \\ gmail.com
}


catarrh as well as pain or a sensation of facial swelling, and impairment or loss of olfaction. An examination of the nasal and oral cavities is crucial. Dental diseases should be excluded. Radiological examination (X-ray images or computed tomography [CT]) is not necessary [2].

The incidence of chronic sinusitis in European countries, according to the GA2LEN study, conducted on 50,000 individuals in 12 countries, is estimated to be $10.9 \%$ [3].

Odontogenic sinusitis (OS) results from infections of the maxillary teeth, trauma to the maxillary teeth, dental procedures, or iatrogenic causes.

Historically, the frequency of OS is estimated to be $10-12 \%$. Currently, the standard for diagnosing $\mathrm{OS}$ is $\mathrm{CT}$ and increasingly widely used cone-beam computed tomography (CBCT). In view of current clinical studies, OS is estimated to account for up to $40 \%$ of all cases of inflammation [4-6].

The types of OS most commonly described in the literature are those associated with oral surgery such as extractions, implants, augmentation of the alveolar process of the maxilla, and sinus lift (SL) procedures [6]. However, it has been shown that pathologies associated with periodontal diseases have the greatest impact on the emergence of inflammation of the mucous membrane of the sinus. This is probably linked to a loss of bone mass in the alveolar process [7].

Pathologies most frequently associated with OS are those of the maxillary second molars, given their location relative to the base of the maxillary sinus [8].

There are significant differences in the microbiology of OS with regard to inflammations of different etiology. In the case of OS, flora of the oral cavity dominates, whereas, in the remaining instances of inflammation, bacteria characteristic of the nasal cavity dominate. It was reported that almost $66.7 \%$ of cases of odontogenic inflammation were caused by anaerobic bacteria [9]. Puglisi et al. believe that all odontogenic inflammation is polybacterial [10]. OS may also have a fungal etiology [11].

One of the most detailed breakdowns of sinus inflammation of odontogenic etiology was presented in the extensive study by Felisati and Chiapasco, which divides OS into three main complication groups: those associated with augmentation of the maxillary sinus, those associated with dental implants, and those associated with oral pathologies and their treatment [12].

In contrast, the dental pathologies that cause sinus inflammation as described in the literature are: dental caries/caries-related pulpitis, periapical granuloma, odontogenic cysts, periodontitis, oro-antral communication, retained roots, unerupted teeth, peri-implantitis, complications after augmentation of the alveolar process, postSL complications, complications after extrusion of a foreign body fragment into the lumen of the maxillary sinus, status post-dental trauma, status post-alveolar process and maxillary trauma, inflammation of the alveolar process of the maxilla after other dental procedures and odontogenic tumors.

In the initial stages of inflammation, lesions are limited to the mucous membrane of the maxillary sinus. There has been no consensus as to what membrane thickness should be considered to be pathological. Currently it is accepted that chronic odontogenic inflammation may be diagnosed when the Schneiderian membrane is thickened $\geq 2 \mathrm{~mm}$, and dental pathologies have been found in the area of the alveolar process $[4,6,13]$.

The gold standard in sinus disease diagnostics is CT, which allows for visualization of the relationship between the teeth and the sinus mucosa in various projections, as well as for an assessment of the ostiomeatal complex, whose patency is a key element for success in the treatment of sinusitis. High-definition (at least 64-slice) CT also gives dentists the ability to track the efficacy of endodontic treatment to the same degree as CBCT [14].

The wide availability of $C B C T$, which is a less expensive technology that requires less time and radiation than $\mathrm{CT}$, has made this diagnostic method more popular. Thus, in the past few years, many authors have leaned toward recognizing CBCT as the gold standard for OS diagnostics [15].

The standard of OS treatment is causal treatment, that is, dental treatment. Until recently it was thought that after the cause was eliminated, pathologies in the sinus would disappear without further action. At present it is estimated that regression of changes affects $30 \%$ of cases, but recent studies have shown that changes in the sinus mucosa do not disappear, even when a therapeutic effect is achieved in the area of a periapical change [16]. In light of the latest studies, treatment should be augmented with pharmacological treatment according to the standards developed for sinus inflammation of different etiology [2].

For OS, surgical treatment is recommended only in cases when dental treatment in combination with conservative treatment is ineffective. A transnasal endoscopic technique is indicated; however, as many researchers have emphasized, some odontogenic diseases necessitate an intraoral approach - for example, odontogenic cysts and oro-antral communication [17].

\section{Material and methods}

This study evaluated 500 patients with a clinical diagnosis of chronic sinusitis and who had CT of the sinuses in the Computed Tomography 
Laboratory of the $4^{\text {th }}$ Military Clinical Hospital in Wrocław between 2013 and 2016. The patients were referred by laryngology, neurology, and maxillofacial surgery specialists.

Patients with a permanent orthodontic appliance were excluded from the study (due to the fact that artifacts can hinder evaluation of the study), as were those with mixed dentition (permanent and milk teeth) and patients who had been referred with a diagnosis other than chronic sinusitis.

Tomography of the sinuses was carried out according to the study protocol. The sinuses' upper limit was established as the superior pole of the frontal sinuses, and the lower limit as the chewing surfaces of the maxillary teeth. The study was conducted with 128-slice Siemens Somatom Emotion Dual computed tomography with 6-mm collimation. For frontal reconstructions, $0.75-\mathrm{mm}$ slices were used.

The CT examinations were independently reviewed by two physicians: one in her third year of specialized training in otolaryngology, and one specialist in dental and maxillofacial surgery. The results were compared with the radiologist's report.

The scans of the frontal, axial, and sagittal cross-sections were reanalyzed in view of the presence of odontogenic cysts, periodontitis, foreign bodies in the sinus, dental implants, oro-antral communication, unerupted teeth, periapical lesions, and the correctness of prior endodontic treatments. These pathologies were assessed separately for each maxillary tooth. The laryngology assessment took into account: the presence of lesions in specific paranasal sinuses with differentiation between wall thickening of the mucosa and lesions involving a larger surface of the sinus; patency of the ostiomeatal complex; the presence of a deviated septum; and issues regarding previous surgical procedures involving the paranasal sinuses.

Analysis of the material collected was done with the program Statistica v. 12. For a statistically significant correlation between and among variables, a test value of $p<0.05$ was accepted. Approval of the Bioethical Committee of the Medical University of Wrocław was acquired (no. KB -696/2017).

\section{Results}

The condition of 500 individuals' dentition (283 women, 217 men, from 13 to 91 years of age [M = 49.2 , SD $=16.9$ years]), for a total of 5484 maxillary teeth, underwent statistical analysis. Bilateral sinusitis was diagnosed in $57.0 \%$ of individuals and unilateral sinusitis in $23.4 \%, 12.4 \%$ in their right sinuses and $11.0 \%$ in their left sinuses.
Based on the presence of mucosal thickening in the right and left maxillary sinus, the patients studied were divided into four subgroups. For the purposes of this work, the following designations regarding involvement of the sinuses were used:

- group 1: No mucosal thickening was noted in any sinuses $(n=98)$;

- group 2: Mucosal thickening was noted in at least one sinus on the right side, including the right maxillary sinus $(n=65)$;

- group 3: Mucosal thickening was noted in at least one sinus on the left side, including the left maxillary sinus $(n=59)$;

- group 4: Mucosal thickening was noted in at least one sinus on both sides, including the maxillary sinuses $(n=277)$;

- group 5: Mucosal thickening involved a sinus/ sinuses other than the maxillary $(n=1)$.

In light of the significant group of patients who showed no sign of changes in the sinus (98 persons), this group was treated as a control in the statistical analysis.

Among the 500 patients with a clinical diagnosis of chronic sinusitis and referred for CT examination, $19.6 \%$ showed no inflammatory changes in the mucosa of the paranasal sinuses. All groups had numerous maxillary teeth missing, ranging from $27.3 \%$ in patients with inflammatory changes in the left-side sinuses up to $33.2 \%$ in patients with no inflammatory changes in the sinuses. Disregarding third molars, the missing teeth most often involved premolars and molars (on the right side, 16.0 to $39.8 \%$; on the left side, 25.0 to $37.0 \%$ ). The pathologies most frequently involved teeth 16 and 27. Periodontitis affected $13.3 \%$ of the patients with no extant inflammatory changes in the paranasal sinuses. Clinical symptoms of advanced periodontitis may be similar to the symptoms of sinus inflammation, which could account for erroneous diagnoses of chronic sinusitis in those patients. Roughly $75 \%$ of patients had a deviated septum. The breakdown of odontogenic changes among the study participants was as presented in Table I.

In the group in question, inflammatory lesions involved at least one of the maxillary sinuses in $80.2 \%$ of the patients, and $8 \%$ of the patients had undergone a sinus operation in the past. Involvement of the other sinuses (ethmoid, sphenoid, and frontal) on the right or left side was significantly more common in the patient group with inflammatory changes in the sinuses on the same side and among patients with bilateral inflammatory changes in the sinuses $(p<0.005)$. In $30.2 \%$ of the patients, the degree of dental tissue damage unambiguously indicated the need for endodontic treatment. Unerupted third molars were found in $11.4 \%$ of the patients. In each of the test groups 
Table I. The breakdown of odontogenic changes among the study participants

\begin{tabular}{|lc|}
\hline Type of odontogenic changes & Percentage \\
\hline Periapical changes & 28.8 \\
\hline $\begin{array}{l}\text { Teeth after incorrectly performer } \\
\text { endodontic treatment }\end{array}$ & 24.2 \\
\hline Unerupted teeth & 18.4 \\
\hline Periodontitis & 12.6 \\
\hline Other dental changes in existing teeth & 11.2 \\
\hline Odontogenic cyst & 8.4 \\
\hline Presence of a foreign body & 6.2 \\
\hline Presence of a retained root & 1.0 \\
\hline Oro-antral communication & 0.8 \\
\hline Implants & 0.4 \\
\hline Other & 2.8 \\
\hline
\end{tabular}

(except group 5), women constituted the majority (50.9-68.4\%). The proportion of changes in patients with dental foci in maxillary teeth on the same side as isolated unilateral inflammation of the maxillary sinus was significantly higher than that on the opposite side $(p<0.001)$, as shown in Figure 1. Odontogenic cysts occurred signifi-

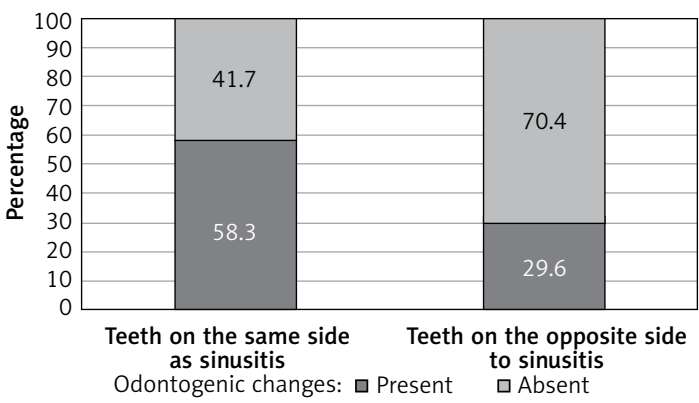

Figure 1. The percentage of patients with isolated single sided maxillary sinusitis in groups with dental foci on the same side as the inflammation of the maxillary sinus and on the other side

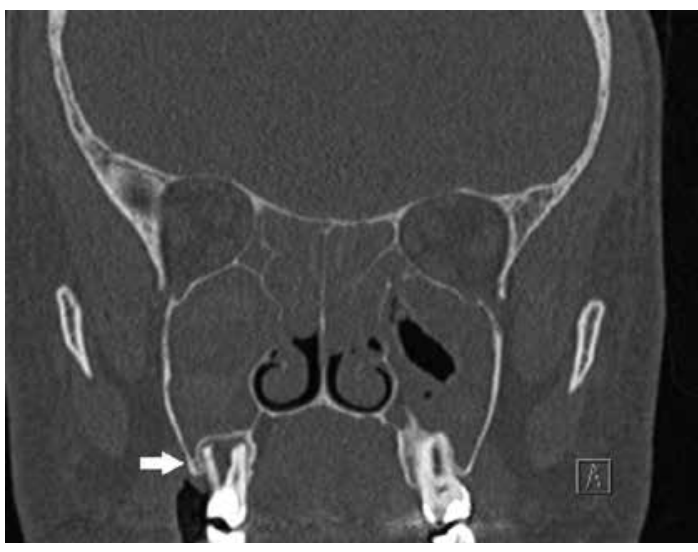

cantly more frequently on the ipsilateral side than inflammatory changes in the maxillary sinus $(p=$ 0.031 ), as seen in Figure 2. They also occurred significantly less frequently in the patient group with no changes in the sinuses than in the group with changes on the left side $(p=0.005)$ and changes on both sides $(p=0.011)$. Foreign bodies in the sinuses occurred significantly more frequently on the ipsilateral side as inflammatory changes in the sinus ( $p=0.041)$. They also occurred significantly less frequently in the patient group with changes on the right side than in the group with no pathological changes in the sinuses $(p=0.027)$. Roots extruding into the lumen of the sinus were significantly less common in patients with no pathological changes in the sinuses than in patients with right-side $(p=0.014)$ and left-side changes $(p=0.010)$. Changes after endodontic therapy of the right maxilla occurred significantly more frequently in patients with inflammatory changes of the sinuses on the right side than in those with inflammatory changes in the sinuses on the left side $(p=004)$ and on both sides $(p=0.010)$. Interestingly, changes after unsatisfactory endodontic therapy of the right maxilla were significantly more common in patients with no pathological changes in the sinuses than in those with bilateral changes $(p=0.001)$ and changes on the left side $(p=0.019)$. Periapical changes in the right maxilla occurred significantly more frequently in patients with bilateral involvement of the sinuses than in other groups $(p=0.006)$. The proportion of no dental changes in the right maxilla was significantly greater in the group of patients with involvement of the left-side paranasal sinuses than in all others $(p<0.005)$.

\section{Discussion}

A majority of the reports available in the Cochrane, Science Direct, and MEDLINE databases assessed the mucous membranes of the maxillary

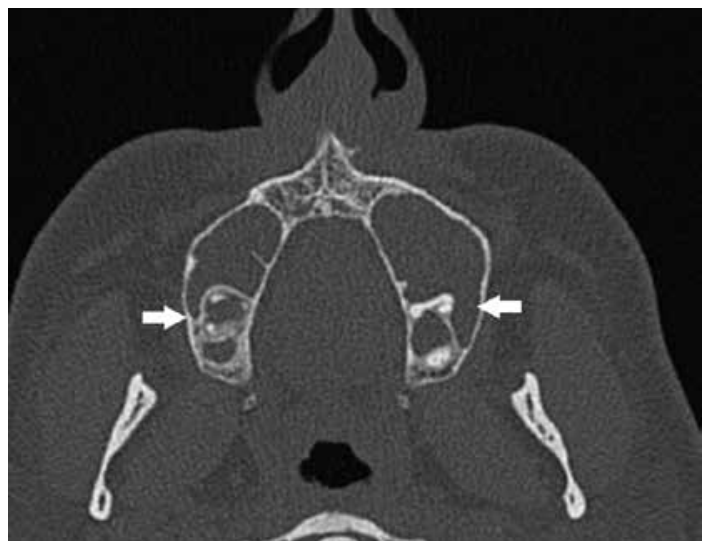

Figure 2. Right maxillary sinus and ethmoid sinuses opacified with inflammatory mucosa. Odontogenic cyst of a right molar and roots of a left molar protruding into the sinus visible (white arrows). Nasal septum without deviation. Ostiomeatal complexes bilaterally opacified 
sinuses in the context of dental therapy that had been performed. In their treatments, Guerra-Pereira et al. evaluated the mucous membranes of the sinuses in 504 CT examinations carried out prior to implant or dental treatment [18]. Shanbhag et al. examined the CBCTs of 243 patients in whom thickening of the sinus mucosa that was greater than $2 \mathrm{~mm}$ had been found with regard to periapical changes and diseases of the periodontium [19]. Janner et al. measured the thickness of the Schneiderian membrane via CBCT in 168 patients being prepared for dental implants [20].

The newest standards of treatment for sinusitis compiled in the European Position Paper On Rhinosinusitis (EPOS) still do not consider a treatment algorithm for patients with existing odontogenic foci [2]. Because of the need for an individual approach in treatment and diagnostics, OS is regarded as an independent disease entity.

Estimates that almost $40 \%$ of chronic sinusitis may be of dental etiology rest mainly on a study group of fewer than 50 individuals or on analyses of clinical materials from laryngology departments supported exclusively by clinical verification $[4,6,21]$. Block and Dastoury assessed the condition of 1,662 sinuses in 831 patients in terms of thickening of the maxillary sinus mucosa and diseased teeth. Thickening of the mucous membrane by more than $2 \mathrm{~mm}$ was noted in 469 maxillary sinuses of 288 patients. In that group, in 210 sinuses, diseased teeth were found [22]. In 1996, Abrahams and Glassberg observed that patients with periodontitis had sinusitis twice as often as patients with no periodontal disease [13]. In their meta-analysis, Arias-Irimia et al. found that in recent years this percentage had dropped significantly; an explanation for this may be the significant improvement in oral hygiene and progress in periodontal and endodontic therapy [23]. OS is most commonly unilateral and has no direct connection to patency of the ostiomeatal complex [4, 24]. Matsumoto et al. concluded that, in a group of 190 patients treated for unilateral sinusitis, $72.6 \%$ of them were odontogenic in origin [25]. In their retrospective work, Longhini and Ferguson noted that in $57 \%$ of cases there was unilateral involvement of the maxillary sinus, and clinical symptoms in the study group occurred from one month to 15 years [24]. On the basis of the group studied in this work, the presence of COS could be identified in $43.2 \%$ of patients with chronic sinusitis.

Current statistical or epidemiological data are limited regarding chronic odontogenic sinusitis in Poland and other European countries [2]. In this study, unilateral sinusitis was diagnosed in $24.8 \%$ of the patients. Odontogenic inflammatory changes were found in $50.8 \%$ of cases of isolated right maxillary sinus inflammation and in $39.0 \%$ of cases of isolated left maxillary sinus inflammation. Patency of the ostiomeatal complexes had no direct effect on the development of OS. Of the 500 patients, 61 individuals (12.2\%) had isolated inflammation of the right maxillary sinus. In that group, right-side dental changes occurred more frequently than did left-side ones. However, in 54 $(10.8 \%)$ of those with isolated inflammation of the left maxillary sinus, dental changes occurred more commonly on the left side than on the right. The proportion of changes in patients with dental foci in maxillary teeth on the same side as isolated unilateral inflammation of the maxillary sinus was significantly higher than that for the opposite side.

The average numbers of existing teeth were similar in the test groups (11 or 12 teeth). However, the fact that, in the test group, the average dentition loss was close to $30 \%$, at an average age of less than 50 years, attests to the extensive tooth loss and the poor state of dentition. There is a lack of epidemiological data for the Polish population regarding dentition in that age group of patients, so it is not possible to assess whether this state of affairs is a reflection of dental problems in patients with chronic sinusitis. The report Oral Health from 2010, commissioned by the European Union Commission on Health, concluded that $41 \%$ of members of the EU population have complete dentition, $32 \%$ have 20 or more teeth, $12 \%$ have $10-19$ of their own teeth, $6 \%$ have $1-9$ teeth, and $7 \%$ suffer from edentulism [26]. No available analyses have assessed tooth loss. However, given the average age in the test group (49.2 years), it should be assumed that most teeth were removed due to inflammation, which could represent a potential primary etiological factor. Thus, in the case of observed tooth loss, especially in the area of the molars and premolars, the possibility of changes of odontogenic etiology in the sinus cannot be excluded.

Depending on the study, considerable differences in percentages arise in the frequency of specific dental pathologies as etiological factors $[21,27]$. Costa et al. cited oro-antral communication as the most common cause of chronic odontogenic inflammation of the maxillary sinus [28], but in the test group examined by Lee and Lee, implants and post-tooth extraction status were most common [29]. Charfi et al. concluded that the most frequent odontogenic cause $(29 \%)$ was periapical changes [30]. In the work of Chemli et al., most frequently it was periapical changes, and among the odontogenic reasons were extruded teeth and oro-antral communications [31]. Račić et al. concluded that odontogenic sinusitis was a complication in $85 \%$ of patients after oral surgery procedures [32]. Guerra-Pereira et al. exam- 

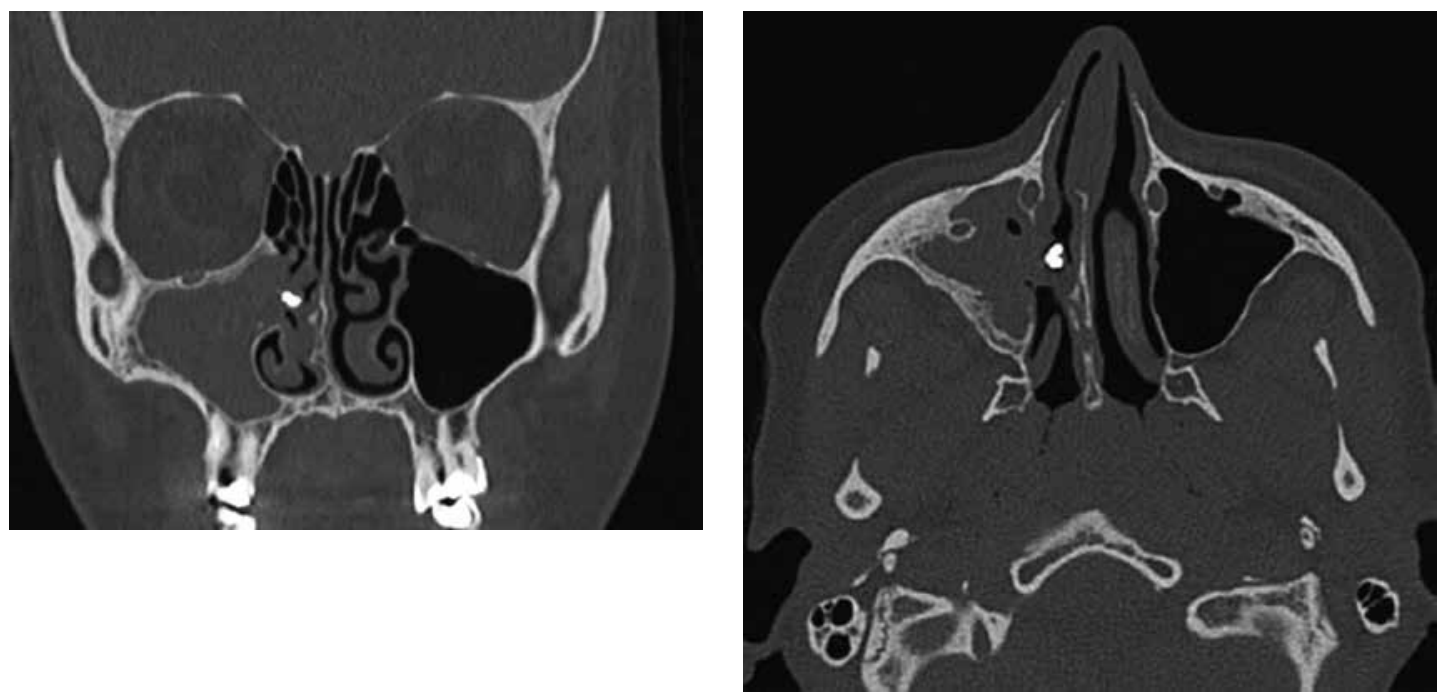

Figure 3. Right maxillary sinus entirely filled with inflammatory mucosa. A foreign body (probably dental material) can be seen between the ostium of the right maxillary sinus and the nasal concha. A slight septum deviation and obstruction of the right ostiomeatal complex are present. Other sinuses are without pathologies

ined 503 CT scans of sinuses of patients prepped for orthodontic or implant treatment. In $25.15 \%$ of the patients in that group - in which dental pathology and corresponding thickening of the sinus mucosa $\geq 2 \mathrm{~mm}$ were observed - teeth after endodontic treatment were the source; in $20.43 \%$, periapical changes were found; in $14.3 \%$, it was the presence of odontogenic cysts; and in $8.25 \%$, there were inflammatory changes of the periodontium [18]. No verification of clinical symptoms was done for these patients. Pokorny and Tataryn selected a group of 67 patients with sinusitis, treated by both laryngologists and dentists, and analyzed their CT examinations with regard to dental foci. In $9 \%$ they found periodontal disease, and in $12 \%$ incorrectly performed endodontic treatment. In $64 \%$ of the cases of odontogenic sinusitis, clear periapical changes were visible [33]. Arias-Irimia et al. identified iatrogenic changes $(55.97 \%)$ as the most frequent cause of OS, as shown in Figure 3. As the remaining etiological factors they identified diseases of the periodontium (40.38\%) and odontogenic cysts (6.66\%) and, in the other $47.56 \%$ of cases, retained roots and complications after tooth extractions [23]. Tröltzsch et al. analyzed radiologic examinations of 173 patients who had undergone surgery for unilateral maxillary sinus inflammation from 2006 to 2013. In 75.0\% of the cases (130 individuals), OS was found. The following odontogenic causes were identified: $64.0 \%$ (83), iatrogenic changes after surgical or dental interventions; $18.0 \%$ (23), periapical changes; $10.0 \%$ (13), periodontitis; $5.2 \%$ (9), post-implant changes; and $2.3 \%$ (4), changes after sinus augmentation [34]. In the test group presented, periapical changes occurred most frequently - in $28.8 \%$ of all those studied.
According to the available literature, the teeth usually responsible for $O S$ are the first molars, then other molars [14, 23, 25, 35]. Maillet et al. assessed 82 CBCT examinations for pathologies of the maxillary sinuses. They concluded that $51.8 \%$ of cases of inflammatory changes in the maxillary sinuses were associated with periapical changes, most frequently involving the second molar [35]. In this dissertation, pathologies most commonly affected teeth 16 and 27.

In conclusion, because this analysis was based exclusively on CT examinations of the sinuses, it had certain limitations. Making an accurate diagnosis requires additional clinical and microbiological testing. For systematized knowledge with regard to OS, wide-ranging studies on a large group of patients are necessary - ideally multi-centered and prospective, if possible. In the face of the possibility of a linkage between sinusitis and teeth that had been removed much earlier on, such analysis would need to be correlated with the condition of the oral cavity and dentition in the populations studied.

\section{Conflict of interest}

The authors declare no conflict of interest.

\section{References}

1. Rosenfeld RM, Andes D, Bhattacharyya N, et al. Clinical practice guideline: adult sinusitis. Otolaryngol Head Neck Surg 2007; 137 (3 Suppl): S1-31.

2. Fokkens WJ, Lund VJ, Hopkins C, et al. European position paper on rhinosinusitis and nasal polyps 2020. Rhinol Suppl 2020; 29: 1-464.

3. Hastan D, Fokkens WJ, Bachert C, et al. Chronic rhinosinusitis in Europe - an underestimated disease. A GA(2) LEN study. Allergy 2011; 66: 1216-23. 
4. Patel NA, Ferguson BJ. Odontogenic sinusitis: an ancient but under-appreciated cause of maxillary sinusitis. Curr Opin Otolaryngol Head Neck Surg 2012; 20: 24-8.

5. Feng L, Li H, Ling-Ling E, Li CJ, Ding Y. Pathological changes in the maxillary sinus mucosae of patients with recurrent odontogenic maxillary sinusitis. Pak J Med Sci 2014; 30: 972-5.

6. Ferguson M. Rhinosinusitis in oral medicine and dentistry. Aust Dent J 2014; 59: 289-95.

7. Sheikhi M, Pozve NJ, Khorrami L. Using cone beam computed tomography to detect the relationship between the periodontal bone loss and mucosal thickening of the maxillary sinus. Dent Res J (Isfahan) 2014; 11: 495-501.

8. Ugincius P, Kubilius R, Gervickas A, Vaitkus S. Chronic odontogenic maxillary sinusitis. Stomatologija 2006; 8: 44-8.

9. Akhlaghi F, Esmaeelinejad M, Safai P. Etiologies and treatments of odontogenic maxillary sinusitis: a systematic review. Iran Red Crescent Med J 2015; 17: e25536.

10. Puglisi S, Privitera S, Maiolino L, et al. Bacteriological findings and antimicrobial resistance in odontogenic and non-odontogenic chronic maxillary sinusitis. J Med Microbiol 2011; 60: 1353-9.

11. Fanucci E, Nezzo M, Neroni L, Montesani Jr L, Ottria L, Gargari M. Diagnosis and treatment of paranasal sinus fungus ball of odontogenic origin: case report. Oral Implantol (Rome) 2013; 6: 63-6.

12. Felisati G, Chiapasco M. Sinonasal complications of dental disease and treatment: prevention, diagnosis, management. 1st ed. Thieme, Stuttgart 2016.

13. Abrahams JJ, Glassberg RM. Dental disease: a frequently unrecognized cause of maxillary sinus abnormalities? AJR Am J Roentgenol 1996; 166: 1219-23.

14. Simuntis R, Kubilius R, Padervinskis $E$, Ryškienè $S$, Tušas $P$, Vaitkus $S$. Clinical efficacy of main radiological diagnostic methods for odontogenic maxillary sinusitis. Eur Arch Otorhinolaryngol 2017; 274: 3651-8.

15. Lofthag-Hansen S, Huumonen S, Gröndahl K, Gröndahl HG. Limited cone-beam CT and intraoral radiography for the diagnosis of periapical pathology. Oral Surg Oral Med Oral Pathol Oral Radiol Endod 2007; 103: 114-9.

16. Nurbakhsh B, Friedman S, Kulkarni GV, Basrani B, Lam E. Resolution of maxillary sinus mucositis after endodontic treatment of maxillary teeth with apical periodontitis: a cone-beam computed tomography pilot study. J Endod 2011; 37: 1504-11.

17. Lawson W, Patel ZM, Lin FY. The development and pathologic processes that influence maxillary sinus pneumatization. Anat Rec (Hoboken) 2008; 291: 1554-63.

18. Guerra-Pereira I, Vaz P, Faria-Almeida R, Braga AC, Felino A. CT maxillary sinus evaluation - a retrospective cohort study. Med Oral Patol Oral Cir Bucal 2015; 20: e419-26.

19. Shanbhag S, Karnik P, Shirke P, Shanbhag V. Association between periapical lesions and maxillary sinus mucosal thickening: a retrospective cone-beam computed tomographic study. J Endod 2013; 39: 853-7.

20. Janner SFM, Caversaccio MD, Dubach P, Sendi P, Buser D, Bornstein MM. Characteristics and dimensions of the Schneiderian membrane: a radiographic analysis using cone beam computed tomography in patients referred for dental implant surgery in the posterior maxilla. Clin Oral Implants Res 2011; 22: 1446-53.

21. Little RE, Long CM, Loehri TA, Poetker DM. Odontogenic sinusitis: a review of the current literature. Laryngoscope Investig Otolaryngol 2018; 3: 110-4.

22. Block MS, Dastoury K. Prevalence of sinus membrane thickening and association with unhealthy teeth: a ret- rospective review of 831 consecutive patients with 1,662 cone-beam scans. J Oral Maxillofac Surg 2014; 72: 2454-60.

23. Arias-Irimia O, Barona-Dorado C, Santos-Marino JA, Martínez-Rodriguez N, Martinez-González JM. Meta-analysis of the etiology of odontogenic maxillary sinusitis. Med Oral Patol Oral Cir Bucal 2010; 15: e70-3.

24. Longhini $A B$, Ferguson BJ. Clinical aspects of odontogenic maxillary sinusitis: a case series. Int Forum Allergy Rhinol 2011; 1: 409-15.

25. Matsumoto Y, Ikeda T, Yokoi H, Kohno N. Association between odontogenic infections and unilateral sinus opacification. Auris Nasus Larynx 2015; 42: 288-93.

26. European Union Commission on Health. Oral health report 2010. Available from: http://ec.europa.eu/commfrontoffice/publicopinion/archives/ebs/ebs_330.

27. Crovetto-Martínez R, Martin-Arregui FJ, ZabalaLópez-de-Maturana A, Tudela-Cabello K, Crovetto-de la Torre MA. Frequency of the odontogenic maxillary sinusitis extended to the anterior ethmoid sinus and response to surgical treatment. Med Oral Patol Oral Cir Bucal 2014; 19: e409-13.

28. Costa F, Emanuelli E, Robiony M, Zerman N, Polini F, Politi M. Endoscopic surgical treatment of chronic maxillary sinusitis of dental origin. J Oral Maxillofac Surg 2007; 65: 223-8

29. Lee KC, Lee SJ. Clinical features and treatments of odontogenic sinusitis. Yonsei Med J 2010; 51: 932-7.

30. Charfi A, Besbes G, Menif D, et al. The odontogenic maxillary sinusitis: 31 cases. Tunis Med 2007; 85: 684-7.

31. Chemli H, Mnejja M, Dhouib M, Karray F, Ghorbel A, Abdelmoula M. Maxillary sinusitis of odontogenic origin: surgical treatment. Rev Stomatol Chir Maxillofac 2012; 113: 87-90.

32. Račić A, Dotlić J, Janosević L. Oral surgery as risk factor of odontogenic maxillary sinusitis. Srp Arh Celok Lek 2006; 134: 191-4.

33. Pokorny A, Tataryn R. Clinical and radiologic findings in a case series of maxillary sinusitis of dental origin. Int Forum Allergy Rhinol 2013; 3: 973-9.

34. Troeltzsch M, Pache C, Troeltzsch M, et al. Etiology and clinical characteristics of symptomatic unilateral maxillary sinusitis:a review of 174 cases. J Craniomaxillofac Surg 2015; 43: 1522-9.

35. Maillet M, Bowles WR, McClanahan SL, John MT, Ahmad M. Cone-beam computed tomography evaluation of maxillary sinusitis. J Endod 2011; 37: 753-7. 